\title{
TWO FINITENESS THEOREMS IN THE MINKOWSKI THEORY OF REDUCTION
}

P. W. AITCHISON

(Received 16 March 1970; revised 16 December 1970)

Communicated by E. S. Barnes

\section{Introduction}

Minkowski proved two important finiteness theorems concerning the reduction theory of positive definite quadratic forms (see [6], p. 285 or [7], $\S 8$ and $\S 10$ ). A positive definite quadratic form in $n$ variables may be considered as an ellipsoid in $n$-dimensional Euclidean space, $R^{n}$, and then the two results can be investigated more generally by replacing the ellipsoid by any symmetric convex body in $R^{n}$. We show here that when $n \geqq 3$ the two finiteness theorems hold only in the case of the ellipsoid. This is equivalent to showing that Minkowski's results do not hold in a general Minkowski space, namely in a euclidean space where the unit ball is a general symmetric convex body instead of the sphere or ellipsoid.

We denote points and vectors in $R^{n}$ as $a, b_{1}$, etcetera, and in particular 0 is the origin, and $u$ denotes a unit vector always. An n-dimensional convex body $K$ is a closed, bounded, and convex subset of a euclidean space, which contains exactly $n$ linearly independent points. In this case, $K$ is symmetric means $K$ is a symmetric set about $0 ; g K$, for a real number $g$, is the set $\{g \mathbf{x} \mid \boldsymbol{x} \in K\}$, and other concepts associated with $K$ such as support plane, width, thickness, boundary, and interior, are as defined in [2] for example, except that "supporting" is used there instead of "support". The distance function $F$ of a convex body $K$ is defined by $K=\{x \mid F(x) \leqq 1\}$, see [5] or [3], and has the following properties:

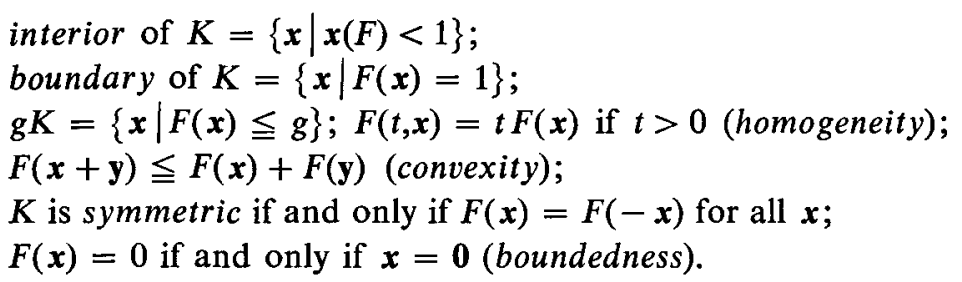

A support plane or support line of $K$ is regular if it intersects $K$ in a single point. 


\section{The Finiteness Theorems}

A lattice $L$ in $R^{n}$ is a set $\left\{\sum_{i=1}^{n} \chi_{i} a_{i} \mid \chi_{i}\right.$ is an integer $\}$ with basis $\left\{a_{1}, a_{2}, \cdots, a_{n}\right\}$ where $a_{1}, a_{2}, \cdots, a_{n}$ are linearly independent. The basis $\left\{a_{1}, \cdots, a_{n}\right\}$ of $L$ is called a $K$-reduced basis of $L$, for an $n$-dimensional convex body $K$, if for each $j$ with $1 \leqq j \leqq n$ we have for integers $p_{i}$

$$
F\left(a_{j}\right) \leqq F\left(p_{1} a_{1}+\cdots+p_{n} a_{n}\right)
$$

whenever g.c.d. $\left(p_{j}, \cdots, p_{n}\right)=1$ (see [7], p. 267, or [5]).

It can easily be shown that when $K$ is an ellipsoid, a $K$-reducd basis of $L$ corresponds to a reduced positive definite quadratic form in $n$ variables (reduced in the Minkowski sense), and two $k$-reduced bases of the same lattic correspond to two equivalent quadratic forms.

Minkowski's finiteness theorems may now be stated as in (I) and (II) (see [6] and [7] for more details).

(I). Let $K$ be a symmetric $n$-dimensional ellipsoid in $R^{n}$ with distance function $F$. There is a finite set $P$ of $n$-tuples of integers, so that if $a_{1}, \cdots, a_{n}$ are linearly independent points satisfying for $j=1, \cdots, n$,

$$
F\left(a_{j}\right) \leqq F\left(p_{1} a_{1}+\cdots+p_{n} a_{n}\right)
$$

whenever $p \in P$ and g.c.d. $\left(p_{j}, \cdots, p_{n}\right)=1$, then $\left\{a_{1}, \cdots, a_{n}\right\}$ is a K-reduced basis.

In other words, if the coefficients of a positive definite quadratic form satisfy a certain finite set of linear inequalities then that quadratic form is reduced in the Minkowski sense.

(II). Let $K$ be an $n$-dimensional ellipsoid in $R^{n}$. Considering all $K$-reduced bases of all $n$-dimensional lattices in $R^{n}$, there are only finitely many different integral unimodular transformations which transform a K-reduced basis again into a K-reduced basis.

In other words, there are only finitely many integral unimodular transformations which can transform a given reduced positive definite quadratic form into an equivalent such form.

One naturally wonders whether the above results on ellipsoids can be generalized to arbitrary convex bodies. For $n=2$, this question was investigated by Minkowski, who proved the first and probably also the second of the following two results. (See [8], p. 193.)

(III). Let $K$ be a symmetric two-dimensional convex body in $R^{2}$ with distance function $F$. If $a_{1}$ and $a_{2}$ are linearly independent, then $\left\{a_{1}, a_{2}\right\}$ is a K-reduced basis if and only if 
and

$$
\begin{aligned}
& F\left(a_{1}+a_{2}\right) \geqq F\left(a_{2}\right), \\
& F\left(a_{1}-a_{2}\right) \geqq F\left(a_{2}\right),
\end{aligned}
$$

$$
F\left(a_{2}\right) \quad \geqq F\left(a_{1}\right)
$$

(IV). Let $K$ be a symmetric two-dimensional convex body in $R^{2}$ with distance function $F$. There are finitely many integral unimodular transformations which transform a K-reduced basis again into a K-reduced basis if, and only if, every support line of $K$ is regular.

However, for $n \geqq 3$ the situation is quite different. Most of the present paper is devoted to the proofs of the theorems (V) and (VI) below, which show that for $n \geqq 3$ the converses of (I) and (II) hold.

(V). For $n \geqq 3$ let $K$ be an $n$-dimensional, symmetric convex body which has the distance function $F$. Suppose there is a finite set $P$ of $n$-tuples of integers satisfying: if $a_{1}, \cdots, a_{n}$ are linearly independent points satisfying, for $j=1, \cdots, n$,

$$
F\left(a_{j}\right) \leqq F\left(p_{1} a_{1}+\cdots+p_{n} a_{n}\right)
$$

whenever $\boldsymbol{p} \in P$ and g.c.d. $\left(p_{j}, \cdots, p_{n}\right)=1$, then $\left\{\boldsymbol{a}_{1}, \cdots, \boldsymbol{a}_{n}\right\}$ is a K-reduced basis. Then $K$ is an ellipsoid.

(VI). For $n \geqq 3$ let $K$ be an $n$-dimensional, symmetric convex body in $R^{n}$. If all K-reduced bases of all $n$-dimensional lattices in $R^{n}$ are considered, then suppose there be only finitely many integral unimodular transformations which transform a K-reduced basis again into a K-reduced basis. Then $K$ is an ellipsoid.

In $\$ 3$ I give a characterization of the ellipsoid which is needed for the proofs of theorems (IV), (V), (VI). As I have been unable to find proofs of theorem (IV) in the literature, I give a proof of it in $\S 4$. In the remainder of the paper I prove (V) and (VI).

\section{A characterisation of the ellipsoid}

We require some properties of the ellipsoid for the proofs to follow. We define the width of a convex body $K$ in the direction $\boldsymbol{u}$ to be the distance between the two support planes of $K$ perpendicular to $\boldsymbol{u}$. Two $n$-dimensional convex bodies $K_{1}$ and $K_{2}$ in $R^{n}$ are called equivalent if the ratio of the width of $K_{1}$ in the direction $u$ to that of $K_{2}$ is constant as $u$ varies in $R^{n}$. We need the following two results, both of which are proved in [1]. (Result (VII) is Theorem 1 of [1], and (VIII) is contained in Lemma 1 of [1].) 
(VII) Let $K$ be a 3-dimensional symmetric convex body in $R^{3}$ with all its support planes regular. Suppose there exists a constant $h$ such that $0<h<1$, and $h$ has the following property. The 2-dimensional convex bodies $W_{1} \cap K$ and $W_{2} \cap K$ are equivalent for all pairs of parallel planes $W_{1}$ and $W_{2}$ which both intersect the interior of $K$ but not the interior of $h K$. Then $K$ is an ellipsoid.

(VIII). Let $K_{1}$ and $K_{2}$ be 2-dimensional convex bodies lying in parallel planes. Suppose that whenever there is a vector $u$ parallel to the plane of $K_{1}$ and $K_{2}$ such that all four support lines parallel to $\boldsymbol{u}$ of $K_{1}$ and $K_{2}$ are regular, then the following property holds. The chord joining the (unique) points of intersection with $K_{1}$ of the two support lines of $K_{1}$ parallel to $u$, is parallel to the corresponding chord of $K_{2}$. Then $K_{1}$ is equivalent to $K_{2}$.

Finally we need the result of (IX) to deal with the difficult cases in (V) and (VI) when there are non-regular support planes.

(IX). Let $K$ be a 3-dimensional symmetric convex body in $R^{3}$ such that no support plane of $K$ intersects $K$ in just a segment. Suppose that for each regular support plane $T$ of $K$ there is a constant $h=h(T)$ (depending on $T$ ) such that $0<h<1$ and $h$ has the following property. If $W_{0}$ is the plane through 0 parallel to $T$ then $W_{0} \cap K$ and $W \cap K$ are equivalent for every plane $W$ which is parallel to $T$ and intersects the interior of $K$ but not that of $h K$. Then all support planes of $K$ are regular.

ProOF OF (IX). By hypothesis a support p'ane of $K$ can intersect $K$ in a sing!e point or a plane face but not a segment. Let $D$ and $D^{\prime}$ be the intersections of two parallel support planes, $T$ and $T^{\prime}$, with $K$, and assume that $D$ (and so by symmetry $D^{\prime}$ ) is a plane face. The aim of the proof is to show that for any vector $a$ parallel to the plane of $D$, there is a plane face of $K$ parallel to $a$ and different from $D$ and $D^{\prime}$. This property is then shown to result in a contradiction.

Let $a$ be any vector parallel to the plane of $D$. Let $U$ be one of the symmetric pair of support planes of $K$ which are parallel to the plane containing $L_{1}$ and $L_{2}$, where $L_{1}$ and $L_{2}$ are support lines of $D$ and $D^{\prime}$ parallel to $a$ and not symmetric about $\mathbf{0}$. There are two cases: (i) $U$ intersects both $D$ and $D^{\prime}$; (ii) $U$ does not intersect $D$ or $D^{\prime}$. ( $U$ cannot intersect just one of $D$ and $D^{\prime}$, because the intersection of $D$ say and $U$ would lie in $L_{1}$, in which case $U$ would also contain $L_{2}$ which intersects $D^{\prime}$.)

In case (i), $U \cap K$ must contain a segment on the boundary of $K$ (joining a point of $D$ and a point of $D^{\prime}$ ), and so by the hypothesis it must contain a plane face of $K$ parallel to $a$ and different from $D$ and $D^{\prime}$.

In case (ii), the plane $U_{0}$ parallel to $U$, through $\mathbf{0}$, clearly intersects the relative interiors of $D$ and $D^{\prime}$. We can choose a plane $U_{1}$ parallel to $U$ and suf- 
ficiently close to $U$ such that $U_{1}$ does not intersect $D$ or $D^{\prime}$ or the interior of $h K$ ( $h$ is the constant $h(U)$ of the hypothesis). Then by hypothesis $U_{1} \cap K$ and $U_{0} \cap K$ are equivalent. The boundary of $U_{0} \cap K$ contains a straight segment parallel to $\boldsymbol{a}$ in its intersection with $D$, and a property of equivalence (see [1], Lemma 2) shows that the boundary of $U_{1} \cap K$ also contains a segment parallel to $a$. Hence there must be a plane face of $K$ parallel to $a$ and distinct from $D$ and $D^{\prime}$ which contains this segment.

In both (i) and (ii) we conclude that there is a plane face of $K$ parallel to $a$ and distinct from $D$ and $D^{\prime}$. However, there are uncountably many such plane faces corresponding to the mutually non-parallel vectors $a$ which are parallel to the plane $T$. Any two such plane faces must be distinct since the only plane faces of $K$ parallel to two such vectors are $D$ and $D^{\prime}$. Yet a convex body cannot have uncountably many plane faces. This contradicts the assumption that $D$ was a plane face of $K$. Hence all support planes of $K$ are regular.

\section{Proof of Theorem IV}

First let every support line of $K$ be regular and $\left\{a_{1}, a_{2}\right\}$ be any $K$-reduced basis of a lattice $L$, so that $F\left(a_{2}\right) \geqq F\left(a_{1}\right)$ by the definition of $K$-reduced basis. If $\left\{b_{1}, b_{2}\right\}$ is any other basis of $L$, then each of $b_{1}$ and $b_{2}$ has the form $p_{1} a_{1}+p_{2} a_{2}$ where $p_{1}$ and $p_{2}$ are integers satisfying g.c.d. $\left(p_{1}, p_{2}\right)=1$. If $\left\{\boldsymbol{b}_{1}, \boldsymbol{b}_{2}\right\}$ is also $K$-reduced, then $F\left(b_{1}\right)=F\left(a_{1}\right)$, and $F\left(b_{2}\right)=F\left(a_{2}\right)$ (a proof of this is contained in [7]; see in particular pp. 278-286). In the first part of the proof we use the above facts to show that $\boldsymbol{b}_{1}$ and $\boldsymbol{b}_{2}$ must be one of a finite set of linear combinations of $a_{1}$ and $a_{2}$, thus showing that there are only finitely many integral unimodular transformations from a $K$-reduced basis of $L$ to another $K$-reduced basis of $L$ (regardless of the choice of $L$.) .

Using the convexity, homogeneity and symmetry of $F$, together with the reduction conditions in (III) we obtain when $p_{1}>0$ and $p_{2}>0$ :

$$
\begin{aligned}
\text { for } p_{1}>p_{2}, F\left( \pm p_{1} a_{1}+p_{2} a_{2}\right) & \geqq p_{1} F\left( \pm a_{1}+a_{2}\right)-\left(p_{1}-p_{2}\right) F\left(a_{2}\right) \\
& \geqq p_{2} F\left(a_{2}\right) ; \\
\text { for } p_{1}=p_{2}, F\left( \pm p_{1} a_{1}+p_{2} a_{2}\right) & =p_{1} F\left( \pm a_{1}+a_{2}\right) ; \\
\text { for } p_{1}<p_{2}, F\left( \pm p_{1} a_{1}+p_{2} a_{2}\right) & \geqq p_{2} F\left( \pm a_{1}+a_{2}\right)-\left(p_{2}-p_{1}\right) F\left(a_{1}\right) \\
& \geqq p_{1} F(a) .
\end{aligned}
$$

In addition, for $p_{1}<p_{2}$ we find using the convexity, homogeneity and symmetry of $F$, together with the reduction conditions, that

$$
\begin{aligned}
F\left( \pm p_{1} \boldsymbol{a}_{1}+p_{2} \boldsymbol{a}_{2}\right) & \geqq p_{2} F\left(\boldsymbol{a}_{2}\right)-p_{1} F\left(\boldsymbol{a}_{1}\right) \\
& \geqq\left(p_{2}-p_{1}\right) F\left(\boldsymbol{a}_{2}\right) .
\end{aligned}
$$

From (1), it follows that $F\left( \pm p_{1} a_{1}+p_{2} a_{2}\right)>F\left(a_{2}\right)$ unless both $p_{2}=1$ and 
$F\left( \pm a_{1}+a_{2}\right)=F\left(a_{2}\right)$. From (2), we find that $F\left( \pm p_{1} a_{1}+p_{2} a_{2}\right)>F\left(a_{2}\right)$ unless $p_{1}=p_{2}=1$. From (4), $F\left( \pm p_{1} a_{1}+p_{2} a_{2}\right)>F\left(a_{2}\right)$ unless both $p_{2}=p_{1}+1$ and $F\left(a_{1}\right)=F\left(a_{2}\right)$, and, from (3), $F\left( \pm p_{1} a_{1}+p_{2} a_{2}\right)>F\left(a_{1}\right)$ unless $p_{1}=1$; hence in this case if $F\left( \pm p_{1} a_{1}+p_{2} a_{2}\right) \leqq F\left(a_{2}\right)$ we must have $p_{1}=1, p_{2}=2$.

If $\pm p_{1} a_{1}+p_{2} a_{2}$ is to be part of a $k$-reduced basis, we need only consider cases where $p_{1}>0$ and $p_{2}>0$, because of the symmetry of $F$ and the fact that $p_{1}=0$, or $p_{2}=0$ can only yield $\pm \boldsymbol{a}_{2}$ or $\pm \boldsymbol{a}_{1}$. It follows from these results and the facts mentioned at the beginning of the proof that the only possible candidates for a $K$-reduced basis of $L$ are

$$
\begin{aligned}
& \pm a_{1} \pm a_{2}, \pm a_{1} \pm 2 a_{2}, \pm a_{1}, \pm a_{2} \text { and } \\
& \pm p_{1} a_{1} \pm a_{2}, \text { where } p_{1} \geqq 2 .
\end{aligned}
$$

In the last case we also found that $F\left(a_{2}\right)=F\left( \pm a_{1}+a_{2}\right)$, and since we also have $F\left(a_{2}\right)=F\left( \pm p_{1} a_{1} \pm a_{2}\right)$ (for appropriate choice of signs), there are three linearly dependent points on the boundary of $K$. This means that $K$ has a nonregular support line, so this last case must be excluded. We are now left with only a finite number of possibilities for points of other $K$-reduced bases, namely

$$
\pm a_{1} \pm a_{2}, \pm a_{1} \pm 2 a_{2}, \pm a_{1}, \pm a_{2} .
$$

There can only be finitely many transformations between bases composed from these points, regardless of the choice of $a_{1}$ and $a_{2}$. This completes the first part of the proof.

Now let us assume that $K$ has a non-regular support line so there is a segment $S$ joining $\boldsymbol{a}$ and $\boldsymbol{b}$ on the boundary of $K$. Choose a sequence of lattices $L_{m}$ with respective bases $\left\{\boldsymbol{a}_{1}^{\boldsymbol{m}}, \boldsymbol{a}_{2}^{\boldsymbol{m}}\right\}$ as follows:

$$
a_{1}^{m}=\frac{1}{m}(b-a), a_{2}^{m}=a .
$$

We now show using the result (III) that $\left\{\boldsymbol{a}_{1}^{m}, \boldsymbol{a}_{2}^{m}\right\}$ is a $K$-reduced basis for all $m$ sufficiently large. We have first of all

$$
F(a) \geqq \frac{1}{m} F(b-a)
$$

for all $m$ sufficiently large, say $m \geqq m_{0}$. Hence by the homogeneity of $F$, we have when $m \geqq m_{0}$

$$
F\left(a_{2}^{m}\right) \geqq F\left(a_{1}^{m}\right) .
$$

Secondly by the convexity, homogeneity and symmetry of $F$, we have

$$
F\left(a_{1}^{m}-a_{2}^{m}\right)=F\left(\left(\frac{m+1}{m}\right) a-\frac{1}{m} b\right)
$$




$$
\geqq\left(\frac{m+1}{m}\right) F(a)-\frac{1}{m} F(b) .
$$

Since $F(a)=F(b)=F\left(a_{2}^{m}\right)=1$, we therefore find for all $m$,

$$
F\left(a_{1}^{m}-a_{2}^{m}\right) \geqq F\left(a_{2}^{m}\right) .
$$

Finally, since $S$ lies on the boundary of $K$ and $a_{1}^{m}+a_{2}^{m} \in S$, we have

$$
F\left(a_{1}^{m}+a_{2}^{m}\right)=1=F\left(a_{2}^{m}\right) \text {. }
$$

Hence, the three conditions of (III) are satisfied and $\left\{a_{1}^{m}, a_{2}^{m}\right\}$ is a $K$-reduced basis of $L_{m}$ when $m \geqq m_{0}$.

Similarly, $\left\{\boldsymbol{a}_{1}^{m}, \boldsymbol{b}\right\}$ is a $K$-reduced basis of $L_{m}$ when $m \geqq m_{0}$. The transformation from the basis $\left\{a_{1}^{m} a_{2}^{m}\right\}$ to the basis $\left\{a_{1}^{m}, b\right\}$ has the matrix

$$
\left[\begin{array}{ll}
1 & m \\
0 & 1
\end{array}\right] \text {. }
$$

Infinitely many of these transformations are different for $m \geqq m_{0}$. Hence, when the boundary of $K$ contains a segment, there are infinitely many integral unimodular transformations which transform a $K$-reduced basis into a $K$-reduced basis of the same lattice.

\section{Proof of Theorem $V$ for $n=3$; regular support planes}

The method of proof in this case is to construct a sequence of lattice bases related to $K$. We show that unless $K$ satisfies conditions which characterize it as an ellipsoid, some of the lattice bases satisfy all of the finite set of inequalities of the hypothesis, yet are not $K$-reduced.

Let $\boldsymbol{u}$ be such that the support plane $U$ with outer normal $\boldsymbol{u}$ is regular, let $U_{0}$ be the plane parallel to $U$ containing 0 , and let $D_{0}=U_{0} \cap K$. We now define a real-valued function $j$ such that if $U^{\prime}$ is the support plane perpendicular to $\boldsymbol{u}$ of the convex body $(j(u) K)$, then $U^{\prime}$ is "considerably smaller" than $D_{0}$. Let $h>0$, be such that: if $U^{\prime} \cap K$ is the support plane of $h K$ with outer normal $\boldsymbol{u}$ then the diameter of $U^{\prime} \cap K$ is equal to $1 / 4$ of the thickness of $D_{0}$. This condition can be satisfied since the diameter of $U^{\prime} \cap K$ must approach zero continuously as $h \rightarrow 1$. This follows because first of all $U^{\prime}$ is a continuous function of $h$ in terms of the usual metric on convex bodies (see [2], p. 133) (this continuity property can be proved using the boundedness of $K$ and the continuity of $F$ ), and secondly because the diameter, as can easily be shown, is a continuous function of $U^{\prime}$. Now define

$$
j(\boldsymbol{u})=\max \left(h, \frac{2}{3}\right), \text { so } j(\boldsymbol{u})<1 .
$$

Because of the convexity and symmetry of $K$, the diameter of $U^{\prime}$ (as defined above) decreases monotonically as $h$ increases. Hence if $U_{1}$ is any plane parallel 
to $U$, not intersecting the interior of $j(u) K$, then the diameter of $\left(U_{1} \cap K\right)$ is less than or equal to $\frac{1}{4}$ of the thickness of $D_{0}$.

Let $U_{1}$ be any plane parallel to $U$ not intersecting the interior of $j(u) K$ and let $D_{1}=U_{1} \cap K$. Let $V_{1}$ and $V_{2}$ be two distinct parallel support lines of $D_{0}$ (in $U_{0}$ ) at the points $b_{1}$ and $\boldsymbol{b}_{2}$. Let $W_{1}$ and $W_{2}$ be distinct support lines of $D_{1}$ (in $U_{1}$ ) at $\boldsymbol{c}_{1}$ and $\boldsymbol{c}_{2}$, which are both parallel to $V_{1}$ and $V_{2}$ and similarly situated with respect to $D_{1}$ and $D_{0}$. (See Diagram 1.)

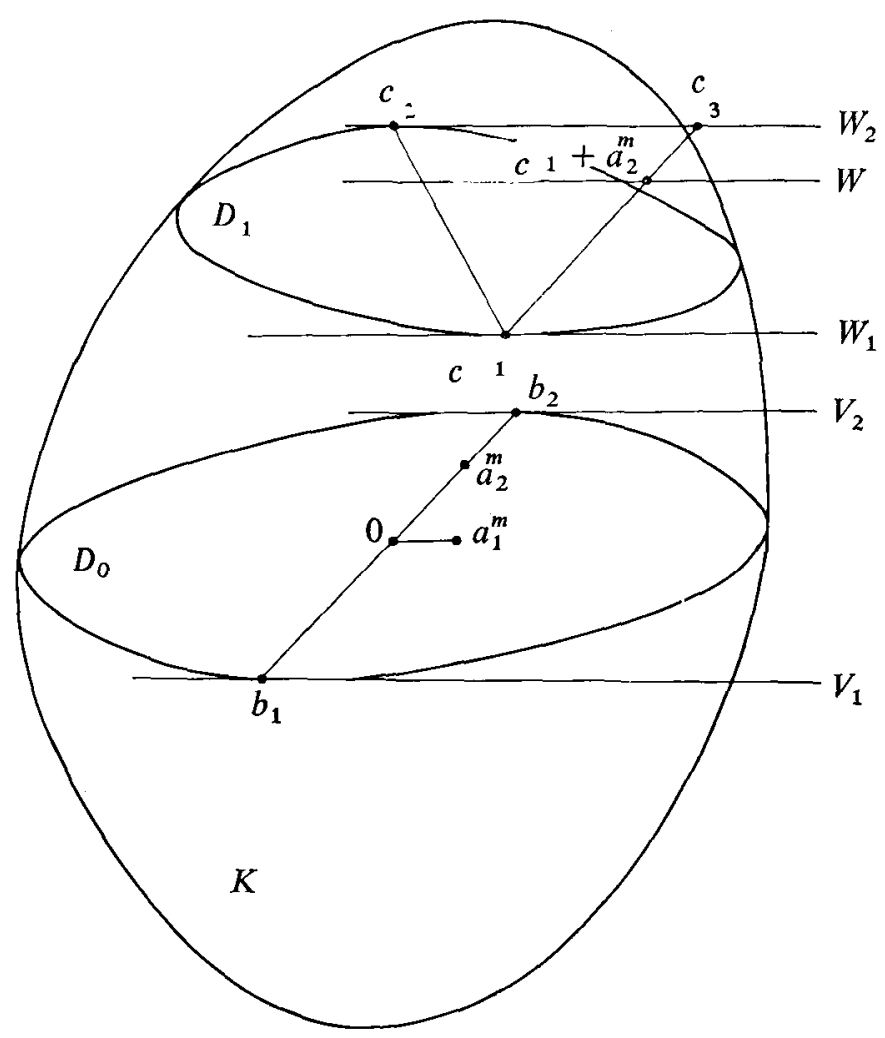

Diagram 1

If the vector $b_{1}-b_{2}$ is parallel to the vector $c_{1}-c_{2}$ for every choice of $V_{1}, V_{2}$, $W_{1}$ and $W_{2}$ when all four are regular support lines, then $D_{0}$ and $D_{1}$ are equivalent by (VIII). It is this result we will eventually obtain.

Assume that for some choice of $V_{1}, V_{2}, W_{1}$ and $W_{2}$, all regular, the vector $b_{1}-b_{2}$ is not parallel to $c_{1}-c_{2}$. We now choose a sequence of lattices $L_{m}$ with respective bases $\left\{a_{1}^{m}, a_{2}^{m}, a_{3}^{m}\right\}$. Notice that $b_{1}=-b_{2}$, and any result concerning the plane $U_{1}$ also applies to the plane $(-1) U_{1}$, by the symmetry of $K$.

Let $c_{3}$ be the point on $W_{2}$ such that the vector $c_{1}-c_{3}$ is parallel to the 
vector $b_{1}-b_{2}$. By assumption $c_{3} \neq c_{2}$ and by the regularity of $W_{2}, c_{3} \notin K$. We define (independently of $m$ )

$$
\boldsymbol{a}_{2}^{m}=k\left(c_{3}-c_{1}\right)
$$

where $k$ is chosen (independently of $m$ ) to satisfy:

(i) $\frac{1}{2}<k<1$; (ii) $c_{1}+a_{2}^{m} \notin K$. Condition (ii) may be satisfied by choosing $k$ sufficiently close to 1 , since $c_{3} \notin K$ and $c_{1}+a_{2}^{m}=k c_{3}+c_{1}(1-k) \rightarrow c_{3}$ a $k \rightarrow 1$. Define $\boldsymbol{a}_{3}^{\boldsymbol{m}}=\boldsymbol{c}_{1}$ (again independent of $m$ ) and

$$
\boldsymbol{a}_{1}^{m}=\frac{k}{m}\left(\boldsymbol{c}_{3}-\boldsymbol{c}_{2}\right) \text {. }
$$

We will now show that all of the finite number of the inequalities (A) of the hypothesis are satisfied by the linearly independent points $a_{1}^{m}, a_{2}^{m}$ and $a_{3}^{m}$ when $m$ is large. First consider $\boldsymbol{a}_{1}^{m}$. Every point of $L_{m}$, which is not a multiple of $\boldsymbol{a}_{1}^{m}$ lies on a line parallel to $V_{1}$, though one of the points $p_{2} a_{2}^{m}+p_{3} a_{3}^{m}$ where the integers $p_{3}$ and $p_{2}$ are not both zero. Since $\boldsymbol{a}_{3}^{m}$ and $\boldsymbol{a}_{2}^{m}$ are defined independently of $m$, these lines have a minimum distance from 0 independent of $m$. Hence for some $q>0$

$$
F\left(\sum_{i=1}^{3} p_{i} \boldsymbol{a}_{i}^{m}\right)>q
$$

whenever $p_{2}$ and $p_{3}$ are not both zero. On the other hand,

$$
F\left(a_{1}^{m}\right)=\frac{k}{m} F\left(c_{3}-c_{2}\right) \rightarrow 0 \text { as } m \rightarrow \infty \text {. }
$$

Hence, there is an $m_{0}$ so that the inequalities (A) hold for $\boldsymbol{a}_{1}^{m}$, when $m \geqq m_{0}$, namely:

$$
F\left(a_{1}^{m}\right) \leqq F\left(p_{1} a_{1}^{m}+p_{2} a_{2}^{m}+p_{3} a_{3}^{m}\right)
$$

for all integers $p_{1}, p_{2}, p_{3}$, whenever $m \geqq m_{0}$.

Consider now the inequalities (A) for $\boldsymbol{a}_{2}^{m}$. We have

$$
\left|a_{2}^{m}\right|=\left|k\left(c_{3}-c_{1}\right)\right|<\left|c_{3}-c_{1}\right| \text {, since } k<1 \text {. }
$$

By our assumption on the widths of $D_{0}$ and $D_{1}$,

$$
\left|c_{3}-c_{1}\right| \leqq \frac{1}{4}\left|b_{2}-b_{1}\right|=\frac{1}{2}\left|b_{2}\right| \text {. }
$$

Hence $F\left(a_{2}^{m}\right) \leqq \frac{1}{2} F\left(b_{2}\right)$, and since $F\left(b_{2}\right)=1$,

$$
F\left(\boldsymbol{a}_{2}^{m}\right) \leqq \frac{1}{2} .
$$

It follows that we need only investigate points $\sum_{i=1}^{3} p_{i} a_{i}^{m}$ in $\frac{1}{2} K$. However $U_{1}$ does not intersect the interior of $j(\boldsymbol{u}) K$ where $j(\boldsymbol{u}) \geqq \frac{2}{3}$, so $F(\boldsymbol{a})>\frac{1}{2} \geqq F\left(\boldsymbol{a}_{2}^{m}\right)$ 
when $a \in D_{1}$. Hence we can restrict our attention to $D_{0}$. We consider points $\left(p_{1} a_{1}^{m}+a_{2}^{m}\right)$, since $p_{3}=0$ in $U_{0}$, and the inequalities (A) only apply to $a_{2}^{m}$ when g.c.d. $\left(p_{2}, p_{3}\right)=1$. We know, by the choice of $c_{3}$, that for some $q>0$

$$
\boldsymbol{b}_{2}=q k\left(c_{3}-c_{1}\right)=q \boldsymbol{a}_{2}^{m} \in V_{2}
$$

By similarity, $q\left(p_{1} a_{1}^{m}+a_{2}^{m}\right) \in V_{2}$. Since $F(a) \geqq 1$ when $a \in V_{2}$,

$$
F\left(q\left(p_{1} a_{1}^{m}+a_{2}^{m}\right)\right) \geqq 1
$$

and so

$$
F\left(p_{1} a_{1}^{m}+a_{2}^{m}\right) \geqq \frac{1}{q}=F\left(a_{2}^{m}\right) .
$$

We have shown that the inequalities (A) hold for $\boldsymbol{a}_{2}^{m}$ and for all $m$, namely: whenever g.c.d. $\left(p_{2}, p_{3}\right)=1$,

$$
F\left(a_{2}^{m}\right) \leqq F\left(p_{1} a_{1}^{m}+p_{2} a_{2}^{m}+p_{3} a_{3}^{m}\right) .
$$

We now consider the inequalities (A) for $a_{3}^{m}$. In this case the conditions $p_{3}=1$, together with $F\left(a_{3}^{m}\right)=1$, restricts our attention to the interior of $D_{1}$. Let $W$ be the line through $\boldsymbol{a}_{2}^{m}+\boldsymbol{a}_{3}^{m}$ parallel to $W_{1}$. All points of $L_{m} \cap U_{1}$ lie on lines parallel to $W_{1}$ through the points

$$
\boldsymbol{a}_{3}^{m}+p_{2} \boldsymbol{a}_{2}^{m}=c_{1}+p_{2} k\left(c_{3}-c_{1}\right) .
$$

When $p_{2}=0$, this line is $W_{1}$ through $c_{1}$; when $p_{2}=1$, it is $W$ through $\boldsymbol{a}_{2}^{m}+\boldsymbol{a}_{3}^{m}$; and when $p_{2}=1 / k$ (not an integer), it is $W_{2}$ through $c_{3}$. Since $W_{1}$ and $W_{2}$ are support lines of $D_{1}$ and by the construction of $k,(1 / k)<2$, the only such line which intersects the interior of $D_{1}$ is $W$. Therefore we need consider only points of $(W \cap K)$. Since $\boldsymbol{a}_{2}^{m}+\boldsymbol{a}_{3}^{m} \notin K$, let $\boldsymbol{c}_{4}$ be the nearest point of $(W \cap K)$ to $a_{2}^{m}+a_{3}^{m}$. The distance between successive lattice points on $W$ is $\left|a_{1}^{m}\right|$, so the number of lattice points between $a_{2}^{m}+a_{3}^{m}$ and $c_{4}$ is at least $\frac{\left|a_{2}^{m}+a_{3}^{m}-c_{4}\right|}{\left|a_{1}^{m}\right|}=\frac{m\left|a_{2}^{m}+a_{3}^{m}-c_{4}\right|}{k\left|c_{3}-c_{2}\right|}=$ Imt say. $t$ is independent of $m$ since $a_{2}^{m}$ and $a_{3}^{m}$ are independent of $m$, and also $t>0$. Hence, all lattice points of $L_{m}$ in $W \cap D_{1}$ must be of the form

$$
p_{1} a_{1}^{m}+a_{2}^{m}+a_{3}^{m}, \text { where }\left|p_{1}\right| \geqq m t .
$$

We have shown therefore that

$$
F\left(a_{3}^{m}\right) \leqq F\left(p_{1} a_{1}^{m}+p_{2} a_{2}^{m}+a_{3}^{m}\right)
$$

for all integers $p_{1}$ and $p_{2}$, except when $p_{2}=1$ and $\left|p_{1}\right| \geqq m t$.

Collecting all the results we have shown that for each $m$ with $m \geqq m_{0}$ and for $j=1,2,3$, 


$$
F\left(a_{j}\right) \leqq F\left(p_{1} a_{1}^{m}+p_{2} a_{2}^{m}+p_{3} a_{3}^{m}\right)
$$

whenever g.c.d. $\left(p_{j}, \cdots, p_{n}\right)=1$, except when $p_{2}=p_{3}=1$ and $\left|p_{1}\right| \geqq m t$. By choosing $m$ large enough, say $m \geqq m_{1} \geqq m_{0}$, we can ensure that none of the $p$, for which the above inequalities fail, lie in the finite set $P$ of the hypothesis. Hence for $m \geqq m_{1}$, the basis $\left\{\boldsymbol{a}_{1}^{m}, \boldsymbol{a}_{2}^{m}, \boldsymbol{a}_{3}^{m}\right\}$ satisfies all of the finite set of the inequalities (A). However $\left\{\boldsymbol{a}_{1}^{m}, \boldsymbol{a}_{2}^{m}, \boldsymbol{a}_{3}^{m}\right\}$ is not $K$-reduced for $m \geqq m_{1}$, since the point

$$
-m \boldsymbol{a}_{1}^{m}+\boldsymbol{a}_{2}^{m}+\boldsymbol{a}_{3}^{m}=\boldsymbol{c}_{1}+k\left(\boldsymbol{c}_{2}-\boldsymbol{c}_{1}\right) \text { for } \frac{1}{2}<k<1
$$

lies in the interior of $\mathrm{K}$ so that

$$
F\left(a_{3}^{m}\right)>F\left(-m a_{1}^{m}+a_{2}^{m}+a_{3}^{m}\right) .
$$

This violates the reduction conditions. Hence we have a contradiction, and so the initial assumption that the vector $b_{1}-b_{2}$, is not parallel to $c_{1}-c_{2}$ must be false. As previously indicated, this leads us to the conclusion that $D_{0}$ is equivalent to $D_{1}$. We have shown, for each initial choice of the regular support plane $U$, that $D_{0}$ and $D_{1}$ are equivalent whenever $U_{1}$ does not intersect the interior of $j(\boldsymbol{u}) K$.

If all support planes of $K$ are regular, then $j(\boldsymbol{u})$ is defined for all $\boldsymbol{u}$, and $j(\boldsymbol{u})<1$. The supremum, $h$ say, of $j(\boldsymbol{u})$ on the closed set of unit vectors satisfies $h<1$. For otherwise, there would be a subsequence $\left\{\boldsymbol{u}_{i}\right\}$ of unit vectors so that $j\left(\boldsymbol{u}_{i}\right) \rightarrow 1$ as $i \rightarrow \infty$ and $j\left(\boldsymbol{u}_{i}\right)>\frac{2}{3}$ for all $i$. If $U_{i}$ is the support plane of $j\left(\boldsymbol{u}_{i}\right) K$ and $D_{i}=U_{i} \cap K$, then the sequence $\left\{D_{i}\right\}$ has a convergent subsequence, according to the Blaschke Selection Theorem (see [2], p. 134), with a plane limit set $D$, and then the plane $W$ of $D$ must be a support plane of $K$. Yet each of the $D_{i}$ must have a diameter no less than $\frac{1}{4}$ of the minimum thickness of any section of $K$ through 0 . Hence $D$ must have a positive diameter, which is impossible, because $W$ is regular. Hence $j(u)$ is bounded above by some constant $h$ with $h<1$, and this $h$ satisfies the hypothesis of (VII), and so $K$ is an ellipsoid.

This completes the theorem for $n=3$, when all support planes of $K$ are regular.

\section{Completion of proof of Theorem $\mathrm{V}$ for $n=3$}

We have to show that every support plane of $K$ is regular, in which case the theorem follows as in $\S 5$. We proved in $\S 5$ (even when some support planes of $K$ are non-regular) that if $\boldsymbol{u}$ is the outer normal of a regular support plane of $K$ and $W_{1}, W_{0}$ are planes perpendicular to $u$ so that $W_{0}$ contains 0 and $W_{1}$ intersects the interior of $K$ but not the interior of $j(\boldsymbol{u}) K$, then $\left(W_{0} \cap K\right)$ and $\left(W_{1} \cap K\right)$ are equivalent. Hence we can apply Theorem (IX) to $K$ to show that all of its support planes are regular, provided no support plane intersects $K$ in just a segment. 
Assume that a support plane $T$ with outer normal $\boldsymbol{u}$ intersects $K$ in just a segment $S$ joining $d_{1}$ and $d_{2}\left(d_{1} \neq d_{2}\right)$. Let $U_{i}, i=2,3, \cdots$ be planes parallel to $T$ at a perpendicular distance of $1 / i$ from $T$. We next define points and lines related to each $U_{i}$ in the same way as we defined points and lines related to $U_{1}$ at the beginning of this proof. Let $U_{0}$ be the plane parallel to $T$ with $\mathbf{0} \in U_{0}$, $D_{0}=U_{0} \cap K$, and, $V_{1}$ and $V_{2}$ be parallel support lines of $D_{0}$ (in $U_{0}$ ). For each $i$, let $D_{i}=U_{i} \cap K$, and let $W_{1 i}$ and $W_{2 i}$ be support lines of $D_{i}$ parallel to $V_{1}$ and $V_{2}$ and respectively, similarly situated about $D_{i}$. Let $d$ and $d_{i}$ be the respective perpendicular distances between, $V_{1}$ and $V_{2}$, and $W_{1 i}$ and $W_{2 i}$. If $V_{1}$ is parallel to the vector $d_{1}-d_{2}$ then clearly $d_{i} \rightarrow 0$ as $i \rightarrow \infty$. Hence if we choose $V_{1}$ sufficiently close in direction to $d_{1}-d_{2}$ yet not parallel to it, then we can find an $i_{0}$ so that $d_{i} \leqq \frac{1}{4} d$ if $i>i_{o}$. We now take this to be the case and further assume that $W_{1 i}, W_{2 i}, V_{1}$ and $V_{2}$ are all regular, which we can do since at most countably many support lines of $D_{i}$ are not regular. Let $\left\{c_{1 i}\right\}=W_{1 i} \cap D_{i}$, $\left\{\boldsymbol{c}_{2 i}\right\}=W_{2 i} \cap D_{i},\left\{\boldsymbol{b}_{1}\right\}=V_{1} \cap D_{0}$, and $\left\{\boldsymbol{b}_{2}\right\}=V_{2} \cap D_{0}$. Finally in addition to the above conditions, we can clearly assume that $b_{1}-b_{2}$ is not parallel to $d_{1}-d_{2}$. $V_{1}$ and $V_{2}$ are now fixed. Note that $U_{i} \cap \operatorname{Int}\left(\frac{2}{3} K\right)$ is empty when $i$ is sufficiently large, say $i>i_{1} \geqq i_{0}$. In this case $D_{i}$, with $i>i_{1}$, now satisfies conditions by which we can show (with $j(u)=\frac{2}{3}$ ) that $c_{1 i}-c_{2 i}$ is parallel to $b_{1}-b_{2}$ in exactly the same way as we previously showed that the vector $c_{1}-c_{2}$ in $D_{1}$ (in the previous notation) is parallel to $\boldsymbol{b}_{1}-\boldsymbol{b}_{2}$ in $D_{0}$. (Even though $j(\boldsymbol{u})$ here does not satisfy the complete condition satisfied by $j(\boldsymbol{u})$ in the previous proof, the condition $d_{i} \leqq \frac{1}{4} d$ is sufficient to follow through the proof for the particular choice of $V_{1}$ and $V_{2}$.)

We now show that the condition that $c_{1 i}-c_{2 i}$ is parallel to $b_{1}-b_{2}$ leads to a contradiction. The sequence $\left\{c_{1 i}\right\}$ clearly has a limit point $d$ in the segment $S$ and we show that $\boldsymbol{d}=\boldsymbol{d}_{1}$ or $\boldsymbol{d}_{2}$. Project all of the sets in question from 0 onto $T$, and denote the projected sets by an asterisk. Then clearly $D_{1}^{*} \supseteq D_{2}^{*} \supseteq \cdots \supseteq S$, and $c_{1 i}^{*} \rightarrow d$ as $i \rightarrow \infty$. Now $W_{1 i}^{*}$ cannot intersect the interior of $S$ since $D_{i} \supseteq S$ and consequently $d=d_{1}$ or $d_{2}$, say $d=d_{1}$. Similarly $c_{2 i} \rightarrow d_{2}$ as $i \rightarrow \infty$. Hence the direction of the vector $c_{1 i}-c_{2 i}$ approaches that of $d_{1}-d_{2}$ as $i \rightarrow \infty$, yet $c_{1 i}-c_{2 i}$ is parallel to $b_{1}-b_{2}$ for $i>i_{1}$ and $b_{1}-b_{2}$, is not parallel to $d_{1}-d_{2}$. This is a contradiction. Hence $T \cap K$ is not just a segment.

Now we can apply (IX) to show all support planes of $K$ are regular. Hence we can prove $K$ is an ellipsoid as in $\S 5$ and the proof of Theorem V is complete for the case $n=3$.

\section{Proof of Theorem $V$ for all $n$}

For $n>3$ we proceed by induction on $n$. Assume that the theorem is true for dimension $(n-1)$ and that $K$ is an $n$-dimensional convex body satisfying 
the hypothesis. Let $U$ be any hyperplane through 0 , and let $J=K \cap U$. Clearly $U$ can be identified with $R^{n-1}$ so that $J$ is an $(n-1)-$ dimensional convex body. Let

$$
P^{\prime}=\left\{\left(p_{1}, \cdots, p_{n-1}\right) \mid\left(p_{1}, \cdots, p_{n-1}, 0\right) \in P\right\} .
$$

We now show that $J$ satisfies the hypothesis of this theorem with $P$ replaced by $P^{\prime}$. Let $a_{1}, \cdots, a_{n-1}$ be linearly independent points in $U$ satisfying for $j=1, \cdots, n-1$,

$$
F\left(a_{j}\right) \leqq F\left(p_{1} a_{1}+\cdots+p_{n-1} a_{n-1}\right)
$$

whenever $\left(p_{1}, \cdots, p_{n-1}\right) \in P^{\prime}$ and g.c.d. $\left(p_{j}, \cdots, p_{n-1}\right)=1$. We must show that $a_{1}, \cdots, a_{n-1}$ is a $J$-reduced basis. Choose a hyperplane $V$ in $R^{n}$, parallel to $U$, which does not intersect the set $F(x) \leqq F\left(a_{i}\right)$ for $x \in R^{n}$, and $i=1, \cdots, n-1$. Define $\mathfrak{a}_{n} \in V$ by

$$
F\left(\boldsymbol{a}_{n}\right)=\min _{\boldsymbol{a} \in \boldsymbol{V}} F(\boldsymbol{a}) .
$$

By the construction of $V$, none of the planes parallel to $V$, through points $p a_{n}$, for an integer $p$, can intersect the sets $F(x) \leqq F\left(a_{i}\right)$, for $i=1, \cdots, n-1$. Hence, for $j=1, \cdots, n$,

$$
F\left(a_{j}\right) \leqq F\left(p_{1} a_{1}+\cdots+p_{n} a_{n}\right)
$$

for all $\left(p_{1}, \cdots, p_{n}\right)$ with $p_{n} \neq 0$. From equations (1) and (2) we find, for $j=1, \cdots, n$,

$$
F\left(a_{j}\right) \leqq F\left(p_{1} a_{1}+\cdots+p_{n} a_{n}\right)
$$

for all $p \in P$, with g.c.d. $\left(p_{j}, \cdots, p_{n}\right)=1$. Since $K$ satisfies the hypothesis of this theorem, it follows that $\left\{\boldsymbol{a}_{1}, \cdots, \boldsymbol{a}_{n}\right\}$ is a $K$-reduced basis. Hence we have, in particular, by the definition of a $K$-reduced basis, for $j=1, \cdots, n-1$,

$$
F\left(a_{j}\right) \leqq F\left(p_{1} a_{1}+\cdots+p_{n-1} a_{n-1}\right)
$$

whenever g.c.d. $\left(p_{j}, \cdots, p_{n-1}\right)=1$. This shows that $\left\{a_{1}, \cdots, a_{n-1}\right\}$ is a $J$-reduced basis.

We have now shown that $J$ satisfies the hypothesis of this theorem, and so by the induction hypothesis, $J$ is an ellipsoid. This result is true for each choice of the hyperplane $U$ through $\mathbf{0}$, so every section of $K$ through 0 is an ellipsoid. It follows from a well-known result, see [4], p. 91, that $K$ is an ellipsoid. Hence the theorem follows for all $n$ by the induction principle.

\section{Proof of Theorem VI}

The proof of this theorem is very similar to the proof of $(\mathrm{V})$ and many of the details of this proof are referred to the proof of $(V)$. We first consider the case where $n=3$. 
Let $U$ be a regular support plane of $K$ and let $U_{0}, j(u), U_{1} D_{0}, D_{1}, W_{1}$, $W_{2}, V_{1}, V_{2}, \boldsymbol{b}_{1}, \boldsymbol{b}_{2}, \boldsymbol{c}_{1}, \boldsymbol{c}_{2}$ and $\boldsymbol{c}_{3}$ be defined as in (V) (see Diagram 2). We assume that, as in (V), for some choice of $V_{1}, V_{2}, W_{1}$ and $W_{2}$, all regular, we have $c_{2} \neq c_{3}$. We now define a sequence of lattices $L_{m}$ with respective bases $\left\{a_{1}^{m}, a_{2}^{m}, a_{3}^{m}\right\}$.

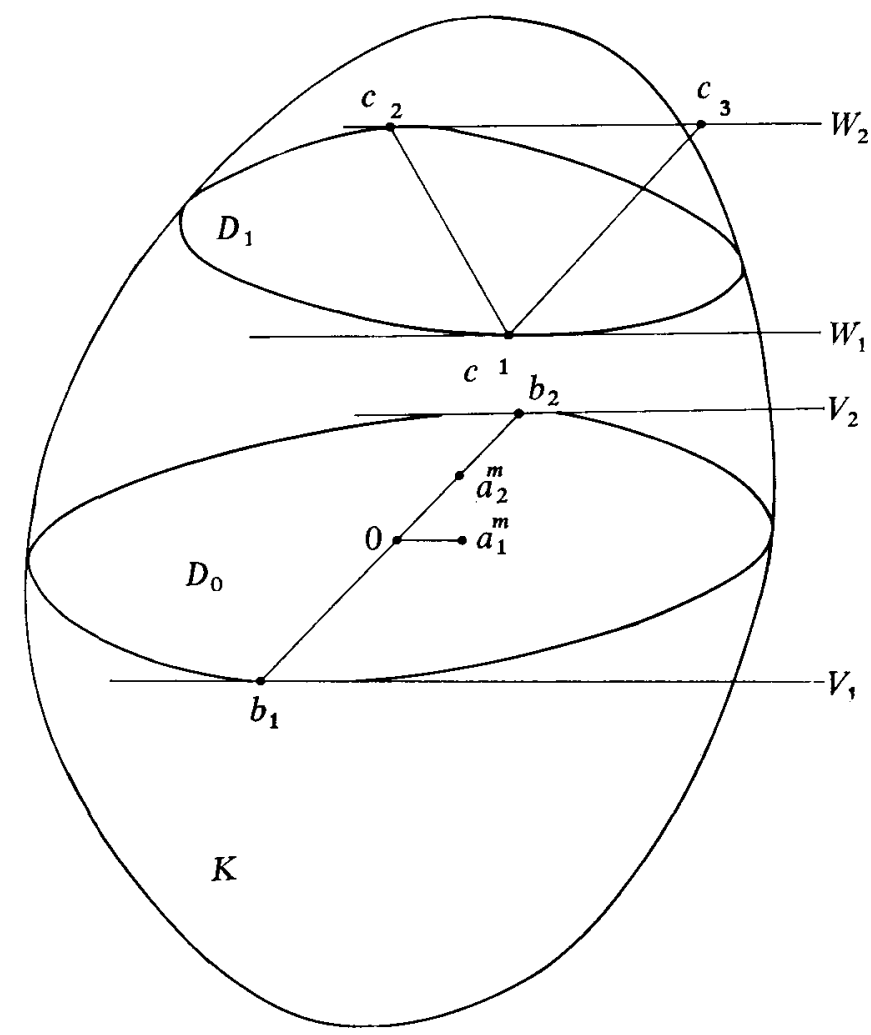

Diagram 2

Define

$$
a_{1}^{m}=\frac{1}{m}\left(c_{2}-c_{3}\right), a_{2}^{m}=c_{3}-c_{1} \text { and } a_{3}^{m}=c_{1} .
$$

The only difference between the situation here and that of $(V)$ is that the line $W$ defined in (V) has now become $W_{2}$. Hence we can show, as in (V). (But without the exceptional points on the line $W$ ), that for $m \geqq m_{0}$ and for $j=1,2,3$,

$$
F\left(a_{j}^{m}\right) \leqq F\left(p_{1} a_{1}^{m}+p_{2} a_{2}^{m}+p_{3} a_{3}^{m}\right)
$$

whenever g.c.d. $\left(p_{j}, \cdots, p_{3}\right)=1$. Hence $\left\{\boldsymbol{a}_{1}^{m}, \boldsymbol{a}_{2}^{m}, \boldsymbol{a}_{3}^{m}\right\}$ is a $K$-reduced basis of $L_{m}$ when $m \geqq m_{0}$. However, $\boldsymbol{c}_{2} \in L_{m}$, since $\boldsymbol{c}_{2}=m \boldsymbol{a}_{1}^{m}+\boldsymbol{a}_{2}^{m}+\boldsymbol{a}_{3}^{m}$, and it is easily 
shown that $\left\{\boldsymbol{a}_{1}^{m}, \boldsymbol{a}_{2}^{m}, \boldsymbol{c}_{2}\right\}$ is also a basis of $L_{m}$. Yet $F\left(c_{2}\right)=F\left(\boldsymbol{a}_{3}^{m}\right)=1$, so $\left\{\boldsymbol{a}_{1}^{m}, \boldsymbol{a}_{2}^{m}, \boldsymbol{c}_{2}\right\}$ is also a $K$-reduced basis of $L_{m}$, for $m \geqq m_{0}$. The transformation from the basis $\left\{\boldsymbol{a}_{1}^{m}, \boldsymbol{a}_{2}^{m}, \boldsymbol{a}_{3}^{m}\right\}$ to the basis $\left\{\boldsymbol{a}_{1}^{m}, \boldsymbol{a}_{2}^{m}, \boldsymbol{c}_{2}\right\}$ has the matrix

$$
\left[\begin{array}{lll}
1 & 0 & m \\
0 & 1 & 1 \\
0 & 0 & 1
\end{array}\right] .
$$

Hence infinitely many of these transformations are different for $m \geqq m_{0}$. This contradicts the hypothesis, so in fact we must have $c_{2}=c_{3}$. As in (V) we deduce $D_{0}$ and $D_{1}$ are equivalent, and that $K$ is an ellipsoid provided no support plane intersects $K$ in a segment.

Assume therefore that the support plane $U$ of $K$ intersects $K$ in a segment $S$ from $\boldsymbol{d}_{1}$ to $\boldsymbol{d}_{2}$. We define $U_{0}, D_{0}, V_{1}, V_{2}, \boldsymbol{b}_{1}$ and $\boldsymbol{b}_{2}$ with $V_{1}$ and $V_{2}$ regular, as in the first part of this proof. We define a sequence of lattices $L_{m}$ with respective bases $\left\{\boldsymbol{a}_{1}^{m}, \boldsymbol{a}_{2}^{m}, \boldsymbol{a}_{3}^{m}\right\}$. Define

$$
a_{1}^{m}=\frac{1}{m}\left(d_{2}-d_{1}\right), a_{2}^{m}=b_{2}, \text { and } a_{3}^{m}=d_{1} .
$$

It is easy to show, as in the first part of the proof of this theorem, that for some $m_{0}$, $\left\{\boldsymbol{a}_{1}^{m}, \boldsymbol{a}_{2}^{m}, \boldsymbol{a}_{3}^{m}\right\}$ is a $K$-reduced basis of $L_{\boldsymbol{m}}$ for $m \geqq m_{0}$. Similarly $\left\{\boldsymbol{a}_{1}^{m}, \boldsymbol{a}_{2}^{m}, \boldsymbol{d}_{2}\right\}$ is a $K$-reduced basis of $L_{m}$ for $m \geqq m_{0}$, and $\boldsymbol{d}_{2}=m \boldsymbol{a}_{1}^{m}+\boldsymbol{a}_{3}^{m}$. The transformation from the basis $\left\{\boldsymbol{a}_{1}^{m}, \boldsymbol{a}_{2}^{m}, \boldsymbol{a}_{3}^{m}\right\}$ to the basis $\left\{\boldsymbol{a}_{1}^{m}, \boldsymbol{a}_{2}^{m}, \boldsymbol{d}_{2}\right\}$ has the matrix

$$
\left[\begin{array}{lll}
1 & 0 & m \\
0 & 1 & 0 \\
0 & 0 & 1
\end{array}\right] .
$$

Infinitely many of these transformations are different for $m \geqq m_{0}$. This contradicts the hypothesis. Hence $U$ cannot intersect $K$ in a segment. As previously noted, this result together with the preceding results leads us to the conclusion that $K$ is an ellipsoid. This completes the discussion of the three-dimensional case.

We now prove the theorem for $n>3$ by an induction proof. Assume that the theorem holds for dimension $(n-1)$, and let $K$ be an $n$-dimensional convex body satisfying the hypothesis of the theorem. Let $U$ be a hyperplane through 0 and let $J=U \cap K$. We now show that the $(n-1)$-dimensional convex body $J$ satisfies the hypothesis of this theorem. 
Let $\left\{a_{1}, \cdots, a_{n-1}\right\}$ and $\left\{b_{1}, \cdots, b_{n-1}\right\}$ be any two $J$-reduced bases of some lattice $L$. Let the unimodular transformation from the first basis to the second one have coefficients $f_{i j}$. We choose a hyperplane $V$ in $R^{n}$ which is parallel to $U$ and does not intersect the sets $F(x) \leqq F\left(a_{n-1}\right)$ and $F(x) \leqq F\left(b_{n-1}\right)$ for $x \in R^{n}$ and define $a_{n}$ by

$$
F\left(a_{n}\right)=\min _{\boldsymbol{a} \in V} F(\boldsymbol{a})
$$

Then, as in the previous proof of (V), $\left\{\boldsymbol{a}_{1}, \cdots, \boldsymbol{a}_{n}\right\}$ and $\left\{\boldsymbol{b}_{1}, \cdots, \boldsymbol{b}_{n-1}, \boldsymbol{a}_{n}\right\}$ are both $K$-reduced bases of the same lattice. The transformation from the first basis to the second has the matrix

$$
\left[\begin{array}{cccc}
f_{11} & \cdots & f_{1, n-1} & 0 \\
\cdot & & \cdot & \cdot \\
\cdot & & \cdot & \cdot \\
\cdot & & \cdot & \cdot \\
f_{n-1,1} & \cdots & f_{n, n} & 0 \\
0 & \cdots & 0 & 1
\end{array}\right]
$$

However, since $K$ satisfies the hypothesis of this theorem, there can only be finitely many of these transformations which are different. Hence only finitely many of the original transformations with the coefficients $f_{i j}$ are different. Therefore $J$ satisfies the hypothesis of this theorem, and so $J$ must be an ellipsoid by the induction hypothesis. It follows as in (V) that $K$ is an ellipsoid, and so the theorem is proved for all $n$ by the induction principle.

\section{References}

[1] P. W. Aitchison 'A Characterisation of the Ellipsoid.' J. Australian Math. Soc. 11 (1970), 385-394.

[2] R. V. Benson, Euclidean Geometry and Convexity (McGraw-Hill, U.S.A., 1966).

[3] T. Bonnesen, and W. Fenchel Theorie der konvexen Körper (Reprint by Chelsea, New York, 1948).

[4] H. Busemann, Geometry of Geodesics (Academic Press, New York, 1955).

[5] J. W. S. Cassels, An Introduction to the Geometry of Numbers (Springer-Verlag, Berlin, 1959).

[6] H.Weyl, 'On Geometry of Numbers'. Proc. Lond. Math. Soc. 47 (1942), 268-289.

[7] B. L. van der Waerden, 'Die Redukionstheorie der positiven quadratischen Formen.' Acta Math. 96 (1956), 265-309.

[8] H. Minkowski, Geometrie der Zahlen (Reprint by Chelsea, New York, 1953).

Department of Mathematics,

University of Manitoba

Winnipeg

Canada 